\title{
Panorama situacional dos cursos de Habilitação em Odontologia Hospitalar: revisão integrativa
}

\author{
Tânia Christina Simões*; Marcelo Lupion Poleti*; Luiz Alberto Valente Soares Júnior**, Gabriela \\ Moura Chicrala***, Paulo Sérgio da Silva Santos****
}

* Doutor (a), Professor (a) do Curso Técnico em Saúde Bucal; Instituto Federal do Paraná, IFPR

** Mestre, Supervisor da Divisão de Odontologia, Hospital de Clínicas, Faculdade de Medicina, USP

*** Doutoranda em Ciências Odontológicas Aplicadas, Faculdade de Odontologia de Bauru, USP

**** Doutor, Professor associado ao Departamento de Cirurgia, Estomatologia, Imaginologia e Radiologia, Faculdade de Odontologia de Bauru, USP

Recebido em 11/04/2019. Aprovado em 22/12/2019.

\begin{abstract}
RESUMO
Cirurgiões-dentistas habilitados em práticas baseadas em evidências científicas e na aplicação de modelos de cuidados em saúde bucal bem-sucedidos trazem benefícios tanto à saúde individual quanto à coletiva. Este estudo traçou um panorama sobre cursos de Habilitação em Odontologia Hospitalar ofertados no Brasil após a regulamentação da Resolução 162/2015 pelo Conselho Federal de Odontologia (CFO), para auxiliar a escolha dos profissionais que pretendem se habilitar na área. O método de pesquisa foi a revisão integrativa da literatura, com análise quantitativa, exploratória e descritiva dos dados. O modelo de busca aplicado foi "habilitação em odontologia hospitalar", na base de dados Google, com uma única consulta em março de 2019. Dezessete (17) propostas foram encontradas. Seguiram para análise treze (13) propostas que informavam número de vagas ofertadas, periodicidade do curso, dias da semana em que ocorriam as atividades, carga horária total, conteúdo programático (teórico e prático) e local da prática. Falta de conteúdo programático $(\mathrm{n}=2)$ e de atualização $(\mathrm{n}=1)$ foram critérios de exclusão imediata. Na análise descritiva das propostas $(\mathrm{n}=10)$ houve exclusões por falta de definição da carga horária teórica e prática $(\mathrm{n}=4)$; informação sobre o conteúdo prático $(n=5)$ e de identificação do local de desenvolvimento e carga horária mínima exigida para a prática. $\mathrm{O}$ estudo evidenciou que, à época da coleta de dados, apenas uma proposta atendia à legislação e à normativa exigidas pelo CFO.

Descritores: Capacitação de Recursos Humanos em Saúde. Cursos. Equipe Hospitalar de Odontologia. Instituições Acadêmicas. Hospitais.
\end{abstract}




\section{INTRODUÇÃO}

O cuidado com o paciente hospitalizado depende da interação do trabalho multiprofissional, resultado da soma de cuidados parciais que se complementam ${ }^{1}$. No entanto, há dificuldade em se estabelecer funções e delegar responsabilidades à equipe multiprofissional. Deste modo, torna-se um desafio coordenar com eficiência uma equipe tão diversificada e especializada de profissionais da saúde; resultando em sobrecarga no processo de gerência de um hospital ${ }^{2}$. Apesar da evidente importância da intervenção odontológica no contexto hospitalar, e de os programas de ação governamental determinarem a participação do cirurgião-dentista nos três níveis de atenção à saúde da população, a presença desse profissional na equipe hospitalar ainda é muito restrita ${ }^{1}$.

O cirurgião-dentista habilitado em Odontologia Hospitalar pode contribuir para a qualidade de vida das pessoas internadas, ficando responsável por cuidados especiais e constantes; não somente para tratar a doença que levou à internação, mas também para cuidar dos demais órgãos e sistemas que podem sofrer algum evento adverso prejudicial à recuperação e ao prognóstico desfavorável do caso. Nesses cuidados devem estar incluídos o tratamento odontológico com a adequação bucal devido à inter-relação entre doenças bucais e sistêmicas. A falta de controle do biofilme dentário em ambiente hospitalar pode acarretar diversas doenças infecciosas, que comprometem a saúde do indivíduo ${ }^{3}$.

A Odontologia Hospitalar visa cuidar das alterações bucais com o objetivo de minimizar o risco de infecção, melhorar a qualidade de vida, reduzir o tempo de internação, adotando um conjunto de ações preventivas, terapêuticas e paliativas em prol da saúde bucal ${ }^{4}$. A preocupação com infecções bucais como foco primário de infecções sistêmicas em pacientes totalmente dependentes de cuidados, internados em Unidades de Terapia Intensiva (UTI), apesar de pouco documentada tem sido relevante nas discussões das equipes interdisciplinares ${ }^{5}$. Medidas para reduzir os focos de infecção de origem bucal incluem cuidados e técnicas locais de higienização, mas também a escolha por medicamentos que auxiliem no equilíbrio do ambiente bucal e na redução da flora bacteriana ${ }^{6,7}$.

Os hospitais contam atualmente com poucos profissionais da área odontológica capacitados a desenvolver cuidados integrais aos pacientes, despertando a comunidade para a importância de habilitar esses profissionais para atuar nos diversos setores de um hospital ${ }^{7}$. Neste contexto, a formação do cirurgião-dentista deve conter em seu currículo questões básicas sobre a atenção integral ao paciente para melhorar a experiência clínica da intervenção odontológica em ambiente hospitalar ${ }^{6}$. A educação dos cirurgiões-dentistas com boas práticas baseadas em evidências científicas e a aplicação de modelos de cuidados de saúde bucal para a gestão de pacientes clinicamente comprometidos podem beneficiar a saúde individual e coletiva ${ }^{5}$.

A participação do cirurgião-dentista na equipe interdisciplinar deve ser vista como positiva para a prevenção e controle de infecções hospitalares, ao realizar, supervisionar e orientar medidas preventivas e curativas em pacientes $\operatorname{críticos}^{5-7}$. A atuação desse profissional em ambiente hospitalar é reconhecida pelo Conselho Federal de Odontologia (CFO) desde 2015. A Resolução CFO 162/2015 reconhece o exercício da Odontologia Hospitalar pelo cirurgião-dentista como habilitação e determina que o curso deve ser realizado com um mínimo de trezentas e cinquenta (350) horas, sendo $30 \%$ de atividades práticas e $70 \%$ de aulas teóricas; que o número máximo de estudantes por turma seja de trinta (30), com, no mínimo, um professor com o título de mestre ou doutor. Além disso, o curso deve ser estruturado com as disciplinas básicas: a) Rotina Hospitalar (gestão, bioética, biossegurança, prontuário, 
prescrição, rounds, prática clínica, segurança do paciente, urgência e emergência); b) Propedêutica Clínica (interpretação de exames, principais agravos, pacientes sistemicamente comprometidos, interações medicamentosas); c) Suporte Básico de Vida (BLS -Basic Life Support) ${ }^{8}$.

No entanto, é raro se deparar com um profissional da área odontológica atuando junto ao corpo clínico da equipe multiprofissional de um hospital. Além disso, poucos estudos na literatura avaliam o impacto positivo da inserção do cirurgião-dentista na equipe multiprofissional que atua na atenção terciária à saúde ${ }^{9}$. Com efeito, um estudo que demonstre a qualidade da formação ofertada para esses profissionais pode reforçar a importância da Odontologia Hospitalar, o impacto financeiro positivo de sua inserção na equipe, assim como a melhora na qualidade de vida dos pacientes $^{9-11}$.

A revisão integrativa é um recurso metodológico que sintetiza o conhecimento advindo das pesquisas disponíveis sobre uma determinada temática e direciona a prática fundamentada em resultados de estudos significativos $^{12}$. Diante deste contexto, o objetivo desta pesquisa foi apresentar um panorama situacional dos cursos de Habilitação em Odontologia Hospitalar, ofertados no Brasil após a regulamentação da Resolução 162/2015 do Conselho Federal de Odontologia, pela revisão integrativa.

\section{METODOLOGIA}

O método de pesquisa utilizado foi a revisão integrativa da literatura. Trata-se de um estudo quantitativo, exploratório e descritivo, no qual a estratégia de busca utilizada foi "habilitação em odontologia hospitalar" no endereço eletrônico www.google.com.br, em uma única busca realizada em março de 2019.

Pela análise dos sítios eletrônicos se constatou que diversas instituições de ensino brasileiras ofertam o referido curso.

Foram incluídos neste estudo os cursos de Habilitação em Odontologia Hospitalar que informavam os seguintes dados disponíveis em seus respectivos sítios eletrônicos: a) número de vagas ofertadas, b) periodicidade do curso, c) dias da semana em que ocorre o curso, d) carga horária total do curso, e) conteúdo programático. Os cursos que não disponibilizaram essas informações mínimas, foram desconsiderados.

Os cursos encontrados no endereço de busca foram salvos como favoritos na barra do Google conforme ordem de aparecimento. Após esta etapa, foram organizados pela ordem de identificação nos seus respectivos endereços eletrônicos. $\mathrm{Na}$ sequência foram organizados por ordem alfabética seguindo os nomes das instituições de oferta e posteriormente categorizados por números ordinais. Os dados coletados foram tabulados com o programa Microsoft Excel® e tratados por estatística descritiva.

\section{RESULTADOS}

Foram inicialmente encontradas dezessete (17) instituições de ensino que ofertam cursos de Odontologia Hospitalar. Após análise das páginas, quatro foram descartadas por se tratar de cursos na forma de ensino a distância (EaD), aperfeiçoamento e/ou capacitação com carga horária reduzida.

As treze propostas de habilitação encontradas foram analisadas e em uma havia apenas informações desatualizadas; outras duas propostas foram descartadas porque não apresentavam conteúdo programático do curso. Para análise descritiva do estudo, foram incluídas dez propostas de habilitação, que informavam os dados que constam no quadro 1 . O número de vagas ofertadas pelo curso foi publicado por apenas cinco instituições. Em relação à periodicidade, apenas uma proposta é ofertada a cada quinze dias; as demais nove são ofertadas mensalmente e a 
Quadro 1. Dados obtidos no endereço eletrônico das instituições com propostas de Habilitação em Odontologia Hospitalar (n=10)

\begin{tabular}{|c|c|c|c|c|c|c|c|}
\hline $\begin{array}{l}\text { Identificação } \\
\text { do curso }\end{array}$ & $\begin{array}{l}\text { Número } \\
\text { de Vagas }\end{array}$ & Oferta & Carga Horária & Duração & $\begin{array}{l}\text { Programação } \\
\text { Teórica }\end{array}$ & $\begin{array}{l}\text { Programação } \\
\text { Prática }\end{array}$ & $\begin{array}{l}\text { Identificação } \\
\text { do Hospital }\end{array}$ \\
\hline 1 & $\mathrm{NI}$ & $\begin{array}{c}\text { Mensal } \\
\text { Sextas-feiras e sábados }\end{array}$ & $\begin{array}{c}420 \text { horas } \\
\text { Teórica (255h) / Prática (105h) }\end{array}$ & 11 meses & Sim & $\mathrm{NI}$ & Sim \\
\hline 2 & 12 & $\begin{array}{c}\text { Mensal } \\
\text { Quintas-feiras a sábados }\end{array}$ & $\begin{array}{c}350 \text { horas } \\
\text { Teórica (260h) / Prática (90h) }\end{array}$ & 9 meses & Sim & $\mathrm{NI}$ & $\mathrm{NI}$ \\
\hline 3 & NI & $\begin{array}{c}\text { Mensal } \\
\text { Segundas a quartas-feiras }\end{array}$ & 480 horas & 18 meses & Sim & Sim & Sim \\
\hline 4 & 24 & $\begin{array}{c}\text { Mensal. } \\
\text { Quintas-feiras a sábados }\end{array}$ & $\begin{array}{c}362 \text { horas } \\
\text { Teórica (246h) / Prática (116h) }\end{array}$ & 11 meses & Sim & $\mathrm{NI}$ & Sim \\
\hline 5 & $\mathrm{NI}$ & $\begin{array}{c}\text { Mensal } \\
\text { Sextas-feiras a domingos }\end{array}$ & $\begin{array}{c}360 \text { horas } \\
\text { Teórica }(70 \%) \text { / Prática }(30 \%)\end{array}$ & $\mathrm{NI}$ & Sim & NI & $\mathrm{NI}$ \\
\hline 6 & 15 a 25 & $\begin{array}{c}\text { Mensal } \\
\text { Sábados e domingos }\end{array}$ & $\begin{array}{c}520 \text { horas } \\
\text { Teórica }(70 \%) \text { / Prática (30\%) }\end{array}$ & 15 meses & Sim & NI & Sim \\
\hline 7 & NI & $\begin{array}{c}\text { Mensal } \\
\text { Quartas a sextas-feiras }\end{array}$ & 384 horas & 12 meses & Sim & NI & NI \\
\hline 8 & $\mathrm{NI}$ & $\begin{array}{c}\text { Mensal } \\
\text { Sextas-feiras a domingos }\end{array}$ & 360 horas & 18 meses & Sim & Sim & Sim \\
\hline 9 & 16 & $\begin{array}{c}\text { Mensal } \\
\text { Segundas a quartas-feiras }\end{array}$ & $\begin{array}{c}384 \text { horas } \\
\text { Teórica }(70 \%) / \text { Prática }(30 \%)\end{array}$ & 10 meses & Sim & Sim & Sim \\
\hline 10 & 20 & $\begin{array}{c}\text { Quinzenal } \\
\text { Quintas-feiras }\end{array}$ & 350 horas & NI & Sim & NI & NI \\
\hline
\end{tabular}

NI - Critério não informado. 
maioria nos finais de semana. Todas as propostas apresentam a carga horária mínima de 350 horas, porém quatro não esclarecem a carga horária das atividades teóricas e práticas. A duração do curso não foi declarada por duas propostas de habilitação. Todas as propostas expõem o seu conteúdo programático teórico, porém apenas quatro identificam o hospital onde serão realizadas as atividades práticas. Destas, apenas três apresentam seu conteúdo programático prático.

$\mathrm{Na}$ síntese qualitativa verificou-se que apenas um curso informou no seu respectivo sítio eletrônico todos os critérios de inclusão com objetividade, apresentando o número exato de vagas ofertadas; a periodicidade do curso com os dias e períodos de aulas; a carga horária total e a distribuição das horas entre as atividades teóricas e práticas, apesar de assinalar as horas por meio de porcentagem, mas em acordo com o previsto na Resolução 162/2015 ${ }^{8}$. O conteúdo programático do curso foi apresentado com as ementas, tanto das atividades teóricas como das atividades práticas; a identificação do hospital onde se desenvolvem as práticas profissionais também foi contemplada. A figura 1 resume as etapas de exclusão dos cursos, apresentadas por meio de fluxograma.

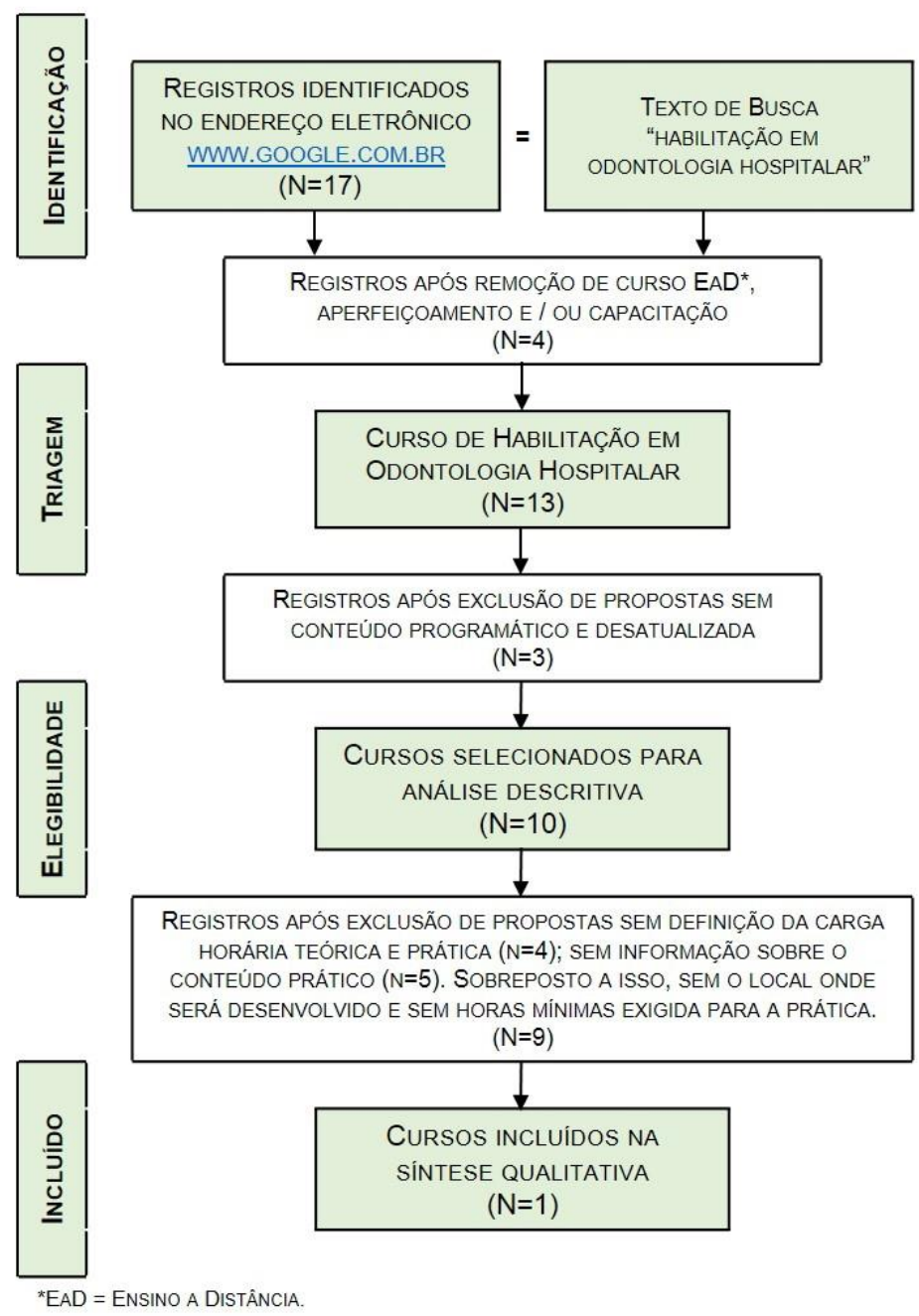

Figura 1. Fluxograma das etapas de busca dos cursos Habilitação em Odontologia Hospitalar 


\section{DISCUSSÃO}

A revisão integrativa é um tipo de pesquisa que permite a síntese de múltiplos estudos publicados e desenvolve conclusões gerais a respeito de uma área de pesquisa apontando falhas no conhecimento que precisam ser corrigidas com a realização de novos estudos ${ }^{9,12}$.

A modalidade de curso Habilitação trata-se de uma especificação de conteúdo associada a uma determinada área de uma graduação. A Resolução 162/2015 regulamenta a habilitação em odontologia hospitalar $^{8}$ e a Resolução 163/2015 define o exercício e a forma de atuação do cirurgião-dentista nos hospitais $^{13}$. O presente estudo evidenciou que diversas propostas analisadas não estão respeitando essas resoluções na íntegra e que há falhas na apresentação dessa modalidade ao público-alvo; dificultando ao cirurgião-dentista a tomada de decisão pelo curso que melhor se enquadra ao seu contexto de vida e ao seu perfil profissional.

A proposta $\mathrm{n}^{\circ} 9$ foi a única que contemplou todos os critérios de inclusão no estudo, que são os preconizados pelo CFO. Mesmo assim não apresenta um cronograma de conteúdo de aulas que comprove as informações divulgadas. Apenas as propostas 6, 8 e 9 apresentam cronograma de dias de aula, porém sem elencar o conteúdo que será ministrado nas referidas datas. Isso poderia ajudar o público-alvo a se organizar com as atividades do curso e também conferir mais transparência às informações sobre a distribuição entre a carga horária teórica e prática a ser desenvolvida no curso.

A apresentação do conteúdo programático foi determinante para as análises, pois mostrou um fato curioso. Apesar de todas as habilitações apresentarem um programa de conteúdo, as propostas se restringem ao conteúdo teórico do curso e com muita variação entre elas. As propostas 1, 4, 5, 6, 7 e 10 indicam apenas o conteúdo teórico em seus respectivos sítios eletrônicos, apesar das propostas 1, 4, 5, 6 indicarem uma carga horária específica para as atividades práticas. Além disso, nas propostas 5, 7 e 10, não há citação do hospital onde serão realizadas as atividades práticas. Isso proporciona um certo desconforto em relação ao cumprimento dessas atividades obrigatórias dentro de um hospital, ambiente crucial para a certificação em Habilitação em Odontologia Hospitalar. A apresentação de um programa prático pelas propostas 3, 8 e 9 favorece a escolha pela habilitação, pois deixa claro que o treinamento será realizado no ambiente hospitalar.

Vale ressaltar que as propostas que relatam a oferta de visita técnica ou monitorada não devem ser consideradas como atividade prática, pois esta modalidade de aprendizagem não permite $o$ atendimento a pacientes em beira leito. Por isso, as alterações feitas pelas resoluções 203 e 204 de 2019 pelo $\mathrm{CFO}^{14,15}$ vieram num bom momento, esclarecendo às competências a serem desenvolvidas pelos profissionais que desejam se habilitar na atenção terciária. Esta informação é recente, e não foi contemplada pela busca, devido ao período em que esta pesquisa foi realizada. Inclusive, as instituições que propõem essa modalidade de ensino estão se adaptando à nova normativa e dessa maneira podem se organizar e apresentar com mais objetividade as suas propostas, evidenciando que proporcionam o desenvolvimento de habilidades e competências necessárias para o manejo adequado de pacientes hospitalizados, que resultem em um atendimento universal, resolutivo, humanizado e de qualidade ${ }^{2,5,16}$.

A habilitação nas especialidades odontológicas está em discussão e especialistas apontam que a presença de cirurgiões-dentistas bem preparados nas equipes multiprofissionais contribui para a melhoria da qualidade de vida dos pacientes ${ }^{17}$. Para isso esses profissionais devem estar preparados para atender em diversos setores da atenção terciária, com conhecimentos sólidos de 
fisiologia, farmacologia e estomatologia, além de conhecimento associado à vocação do hospital em que trabalha, para auxiliar de forma direta na diminuição de custos e na média de permanência do paciente hospitalizado ${ }^{18}$.

A Resolução da Diretoria Colegiada (RDC) no 07 da Agência Nacional de Vigilância Sanitária (ANVISA) de 2010 obriga hospitais a ter em plantão, nas Unidades de Tratamento Intensivo, um cirurgião-dentista para acompanhar os pacientes e coordenar os procedimentos de adequação bucal ${ }^{19}$. O profissional necessita ter uma amplitude de visão e atuação sobre o nível de responsabilidade e do trabalho em equipe multidisciplinar. Para tal feito o cirurgião-dentista deve investir em sua capacitação e expertise ${ }^{17,18}$, desafios que serão superados se for habilitado com práticas baseadas em evidências científicas e com aplicação de modelos de cuidados em saúde bucal bem-sucedidos ${ }^{5}$.

\section{CONCLUSÃO}

O estudo mostrou que apenas uma proposta atende à legislação e à normativa exigidas pelo CFO. A falta de informações nos endereços eletrônicos das propostas avaliadas dificulta a escolha do público-alvo quanto à opção que melhor se enquadra ao seu contexto de vida e ao seu perfil profissional.

\section{ABSTRACT \\ Situational overview of the courses of Qualification in Hospital Dentistry: integrative review}

Dentists qualified on evidence-based practices and on the application of successful oral health care models are beneficial to individual and collective health. The current study presents an overview of Hospital Dentistry Qualification courses available in Brazil after the regulation of Resolution 162/2015 by the Conselho Federal de Odontologia (CFO Brazil), which focuses on assisting the selection by professionals who intend to qualify in this field. Integrative literature review, with quantitative, exploratory and descriptive data analyses, was the research method adopted in the present study. The mesh "hospital dentistry qualification" was used in the single search conducted on Google database, in March 2019. Seventeen (17) proposals were found. Thirteen (13) proposals that included information about vacancies available for students, course periodicity, weekdays when the course took place, total workload, program content (theoretical and practical) and place of practice were selected for analysis. Lack of program content $(n=2)$ and outdated information $(n=1)$ were immediate exclusion criteria. The descriptive analysis applied to the proposals $(n=10)$ has shown that courses were excluded due to lack of definition of the workload of theoretical and practical activities $(n=4)$; as well as of information about the practical content of the course $(n=5)$, the hospital where the practice would take place and the minimum workload required for the practice. One (01) qualification proposal was included in the research based on qualitative synthesis. The current study has shown that one proposal was in compliance with the legislation and regulations required by $\mathrm{FCD}$, as well as that the presentation of the information available in the website about the proposal makes it hard for the target audience to make the option for the course that would best meet their needs.

Descriptors: Health Human Resource Training. Courses. Dental Staff, Hospital. Schools. Hospitals.

\section{REFERÊNCIAS}

1. Rocha AL, Ferreira EF. Odontologia hospitalar: a atuação do cirurgião dentista em equipe multiprofissional na atenção terciária. Arq Centro Estud Curso Odontol. 2014; 50(4):154-60.

2. Shinkai RSA, Del Bel Cury, AA. O papel da odontologia na equipe interdisciplinar: contribuindo para a atenção integral ao idoso. Cad Saúde Pública. 2000; 16:1099-109.

3. Rodrigues ALS, Malachias RC, Da Fonseca Pacheco CM. A importância da saúde bucal em pacientes hospitalizados: uma revisão. Rev Odontol UNICID. 2018; 29(3):243-8.

4. Duarte F, Custodio MM, Ozelin AA, Rossato 
PH, Simões TC, Poleti ML, Sangiorgio JPM. A importância do técnico em saúde bucal na odontologia hospitalar: relato de experiência. Rev Eletr Acervo Saúde [Internet]. 2018 [Acesso em 25/03/2019];17(e57). Disponível em: https://acervomais.com.br/index.php/ saude/article/view/57.

5. Costa JRS, Santos PSS, Torriani MA, Koth VS, Hosni ES, Alves EGR, Arrieira ICO, Miguens Jr SAQ. A odontologia hospitalar em conceitos. Rev AcBO. 2016; 25(2):211-8.

6. Marin C, Bottan ER, Maçaneiro CAR. Visão de profissionais da saúde sobre a inserção do cirurgião-dentista no ambiente hospitalar. Rev Pesq Saúde. 2015;16(1):24-5.

7. Santos TB, Amaral MA, Peralta NG, Almeida RS. A inserção da Odontologia em Unidades de Terapia Intensiva. J Health Sci. 2017; 19(2):838.

8. Conselho Federal de Odontologia (Brasil). Resolução no. 162, de 03 de novembro de 2015. Reconhece o exercício da Odontologia Hospitalar pelo cirurgião dentista. Diário Oficial da União 16 nov 2015; Seção 1.

9. Godoi APT, Francesco AR, Duarte A, Kemp APT, Silva-Lovato CH. Odontologia hospitalar no Brasil. Uma visão geral. Rev Odontol. UNESP. 2009; 38(2), 105-9.

10. Afonso AC, Silva I. Qualidade de vida relacionada com saúde oral e variáveis associadas: revisão integrativa Psic Saúde \& Doenças. 2015; 16(3), 311-30.

11. Mattevi GS, Figueiredo DR, Patrício M, Silva Rath IB. A participação do cirurgião-dentista em equipe de saúde multidisciplinar na atenção à saúde da criança no contexto hospitalar. Ciênc Saúde Colet. 2011;16:4229-36.

12. De Souza MT, Da Silva MD, De Carvalho R. Revisão integrativa: o que é e como fazer. Einstein. 2010; 8(1):102-6.

13. Conselho Federal de Odontologia (Brasil). Resolução no .163 , de 09 de novembro de 2015.
Conceitua a Odontologia Hospitalar e define a atuação do cirurgião-dentista habilitado a exercê-la. Diário Oficial da União, Brasília, 25 nov. 2015, seção 1, p. 120.

14. Conselho Federal de Odontologia (Brasil). Resolução no 203, de 23 de maio de 2019. Altera a Resolução CFO - 162/2015. Diário Oficial da União, Brasília, 23 maio 2019, seção 1, p. 77.

15. Conselho Federal de Odontologia (Brasil). Resolução no 204, de 23 de maio de 2019. Altera as alíneas do art. $2^{\circ}$ da Resolução CFO 163/2015. Diário Oficial da União, Brasília, 23 maio 2019, seção 1, p. 77.

16. Lucas BB, Rodrigues Vieira Júnior JL, Besegato JF, Caldarelli PG. Ensino da Odontologia Hospitalar no Sul do Brasil. Rev ABENO. 2017; 17(2):68-75.

17. Os desafios da habilitação em Odontologia Hospitalar: tratamento odontológico melhora comprovadamente o quadro clínico dos pacientes. Jornal da ABO 2015; 22(151): 12-3.

18. Dos Passos Rodrigues G, Durigon GS, Lisboa ML, Bodanezi, Barros BAC, Duque TM, Munhoz EA, Rath IBS, Camargo AR. Impacto de um projeto de extensão sobre a formação discente para atuação em ambiente hospitalar. Rev Eletr Extensão. 2018; 15(31):67-78.

19. Resolução $\mathrm{n}^{\circ} 7$, de 24 de fevereiro de 2010. Dispõe sobre os requisitos mínimos para funcionamento de Unidades de Terapia Intensiva e dá outras providências. Diário Oficial da União (Seção 1) 2010; 37:48-51.

\section{Correspondência para:}

Tânia Christina Simões e-mail: tania.simoes@ifpr.edu.br Rua João XXXIII, 600 Jardim Dom Bosco 86060-370 Londrina/PR 